\title{
An Educational Program about Nursing Managers' Transformational Leadership, Conflict management Styles and Decision Making Effectiveness
}

\author{
Reda Abd El-Fatah SaidAhmed Abo Gad \& Safaa Mohamed El-Demerdash \\ Nursing Administration Department, Faculty of Nursing, Tanta University, Egypt
}

\begin{abstract}
Background: Within nursing, there are leaders at many levels, such as those in direct leadership roles at a unit level and those in higher leadership roles at a service level. This can often lead to conflict and control-seeking, especially when final decisions need to be made. The study aims to enhance nursing managers' transformational leadership, conflict management styles and decision making effectiveness through an educational program. Quasi experimental design was used. The study was conducted at Medical Center at Tanta city. The sample consists of all available nursing managers (20) pull from different Ministry of Health Hospitals in El-Gharbia. The data was collected by using(1) Knowledge questionnaire test, (2) Transformational leadership scale,(3) Conflict management styles scale and, (4) Decision making effectiveness scale.
\end{abstract}

Results: levels of nursing managers' total knowledge and all items of transformational leadership, conflict management, and decision making were significant improved post than pre program at $P<0.05$. The was significant positive correlation at $p \leq 0.05$ between nursing managers' total level of knowledge and conflict management strategies, decision making effectiveness as well as four dimensions of transformational leadership.

Conclusion: Overall the evidence evaluating the effectiveness of nursing managers training program for enhancing their transformational leadership, conflict management styles and, decision making effectiveness indicates that their knowledge and skills was generally poor pre program. After implementation of the program, their knowledge scores significantly improved and their skills positively changed. It was recommended that early training, especially during undergraduate studies, is absolutely essential for an integrated knowledge of all aspects of leadership, decision making process and a constructive conflict resolution process.

Key word: Nursing Managers, Transformational Leadership, Conflict management style, Decision making effectiveness

\section{Introduction}

Transformational leadership can be practiced in hospitals (Roussel \& Swansburg, 2009). A transformational leader is a person who stimulates and inspires (transform) followers to achieve extraordinary outcomes (Robbins \&Coulter, 2007). He/she pays attention to the concern and developmental needs of individual followers; changes followers' awareness of issues by helping them to look at old problems in a new way; and he /she is able to arouse, excite and inspires followers to put out extra effort to achieve group goals (Warrilow, 2012). Transformational leadership is viewed as the most effective model of leadership because, while it recognizes the importance of rewards, it goes further to satisfy the higher needs of the follower by engaging this person emotionally and intellectually (Surakka, 2008). This leadership behavior aims at the "transformation" of subordinates' needs to higher order needs of the organization through leaders' instilling of his or her goals. A leader motivates his/her subordinates to spend extra effort, to perform beyond expectations and to accomplish the organizational goals via convincing communication of a common vision. So, the subordinates adopt the mission, goals and strategies of the leader and the organization, respectively (Bass $\boldsymbol{\&}$ Avolio, 1999).

Transformational leadership is divided into four areas which embrace: Idealized influence, Inspirational motivation, Intellectual stimulation and Individualized consideration Warrilow (2012) \& Hayati et al., (2014). First; Idealized influence builds confidence, admiration, respect and trust (Bass et al., 2003), providing employees with a sense of mission (Northouse, 2010). For this to occur, nurse leaders need to be role models who their staff seeks to emulate (Ilies et al., 2012). Second; inspirational motivation, the leader communicates a vision, shows enthusiasm, provides an optimistic view towards the future and demonstrates confidence that all goals can be achieved and that goal achievement changes the future positively. Additionally, he/she encourages her/his subordinates to consider emerging problems as challenge and chance. Third; intellectual stimulation: The leader encourages her/his subordinates to question the status quo, and to look at problems from different angles; he/she appreciates intellect and new and creative ways of thinking, and fosters 
subordinates' willingness to change. Finally; individualized consideration, the leader supports his/her subordinate in her/his development by providing learning opportunities. He/she also shows empathy, recognizes and respects individual needs (Bass et al., 2003).

Within nursing, there are leaders at many levels, such as those in direct leadership roles at a unit level and those in higher leadership roles at a service level. This can often lead to conflict and control-seeking, especially when final decisions need to be made (Casida \& Parker, 2011). Wilmont \& Hocker (2001) defined conflict as a struggle or contest between people with opposing needs, ideas, beliefs, values, or goals. Hendel, et al, (2005) reported six areas that cause conflict within nursing: defiant behavior; stress; space; doctor authority; beliefs, values and goals; and others.

Leaders interfere with the organizational conflicts directly through management styles which involving (avoidance, accommodating, compromising, competing and cooperating) that will have impacts on controlling the conflicts. Conflict management is a process of identifying the appropriate roles of conflict among groups and applying the techniques effectively to resolve it for the sake of organizational efficacy (Fakhimi, 2004). The ability to creatively manage internal conflict in the organization is becoming essential requirement (Hendel, et al., 2005) Leadership and choice of conflict management strategies can strongly influence outcomes of a conflict and decisions (DeChurch \& Marks, 2001).

Nursing leaders are frequently confronted with difficult decisions, ranged from strategic decisions through to managerial decisions and routine operational decisions, particularly in financial management, resource allocation, activity planning, and budget monitoring and control (Allred et al., 1995). As well as they do not make decisions in a vacuum. Organizational culture, top managerial support, management team characteristics, decision-specific characteristics and, even adequate staffing can affect decision making (Shirey, 2009). Time constraints encourage nurse managers to make decisions very quickly (Clark-Burg, 2013). Decision-making is the process of establishing criteria by which a nurse leader can develop and select a course of action from a group of alternatives (Carroll, 2008). Decision-making is a management skill that includes the process leading up to the final decision. Effective decision making is one of the attribute of an efficient leader (Tatum, et al., 2003). Effective decision making is defined as the process through which alternatives are selected and then managed through implementation to achieve objectives (Jasmin, 2007).

Effective decisions result from a systematic process, with clearly defined elements, that is handled in a distinct sequence of steps in the sense that, in most decision situation, managers go through a number of steps that help them think through the problem and develop alternative solutions (Jasmin, 2007). A manager might use several methods to make an effective decision. It includes decision by authority without group discussion, expert, averaging individuals' opinions, authority after group discussion, minority, majority vote, and by consensus (Johnson and Johnson, 2000).

\section{Significance of study}

Nurses are constantly involved in the decision-making which can be involves some conflicts or dissatisfaction. Whether as a staff nurse or a manager, regardless of the practice setting .Effective decisionmaker makes decisions with competence and confidence. Identifying and using the decision-making process help managers to make more effective decisions. The dynamic and uncertain nature of health care environment requires nursing managers to be competent decision makers in order to respond to clients' needs. Moreover, changes in patient needs, medical technology, and financial resources create uncertainty in health care organizations' and require redesign of its structure and its process of care. The role and influence of transformational leadership are becoming increasingly important in today's complex and continually changing health care organizations.

\section{Aim of the study}

The study aims to enhance nursing managers' transformational leadership, conflict management styles and decision making effectiveness through an educational program.

\section{Research question}

Does an educational program will enhance nursing managers' transformational leadership, conflict management styles and decision making effectiveness?

\section{Design:}

\section{Materials and Method}

Quasi experimental design was used

Setting:

The study was conducted at Medical Center at Tanta city, which affiliated to The Ministry of Health in El-Gharbia. That was pull nursing managers from different hospitals in El-Gharbia 


\section{Subjects:}

The sample consisted of twenty nursing managers working in the above mentioned setting and willing to participate in the study.

Tools: To achieve the aim of the present study four tools were used

\section{Tool I: Knowledge Questionnaire Sheet}

This tool was designed to collect data from nursing managers for the purpose of assessing their knowledge about transformational leadership, conflict management style and decision making process. The questions were constructed in either form of true \& false, cross matching, multiple choices, or complete. The tool was developed by the researchers after reviewing of related literatures (Clark-Burg, (2013); James \& Ogbonna (2013); Harvey \& Technical Information Service, (2007). The tool consisted of two parts as follows

\section{Part one}

The first part for data pertaining characteristics of the study subjects such as age, years of experience, years of experience as nursing managers and position

\section{Part two}

The second part of the tool contained (40) questions grouped under three headings as follow: transformational leadership (11questions), conflict management (16 questions), and decision making (13 questions).

\section{Scoring system}

The question was scored by one for each correct answer and zero for incorrect answer the total score was 40 grades. Scoring represent varying levels of nurses knowledge ranging from good knowledge (85-100), fair knowledge $(85<65)$, and poor knowledge $(<65)$ points.

Tool II: Transformational leadership scale by Kalf (2010) based on Bass \& Avolio (1999). It was used for the purpose of assessing nursing managers' transformational leadership behavior. It consisted of 33 items are grouped into four subscales: idealized influence has 8 items, intellectual simulation has 12 items, inspirational motivation has 8 items and, individualized consideration 5 items.

\section{Scoring system}

The subject's responses were in five points Likert scale ranging from (5-1) always (5), often (4), sometimes (3), rarely (2) and never (1). The maximum scores of these scale were 165, these scores were classified into three categories according to the following strength: high $75-100 \%$, moderate $60-<74 \%$ and, low $<60 \%$,

Tool III: Conflict management style scale by Rahim and Magner, (1995). This questionnaire was applied to identify nursing managers' conflict styles and examine how it varies in different contexts or relationships. It consists of 25 items.

\section{Scoring system}

The subject's responses were in five points Likert scale ranging from (5-1) $5=$ always, $4=$ often, $3=$ sometimes \& $1=$ never. Scores from 21 to 25 are representative of a very strong style, 15 to 20 are representative of a strong style, 11 to 15 are representative of an average style, and 6 to 10 are representative of a weak style and 0 to 5 representative of a very weak style.

Tool IV: Decision making effectiveness scale. This tool was developed by the researchers and guided by abahe, (2011) and El-Ghazali (2012). It consists of 39 items, 10 factors affect administrative decision making in health care organizations, 10 items for best ways of administrative decision making in health care organizations, 10 items for problems that affect administrative decision making in health care organizations and 9 the effectiveness of the decision-making.

\section{Scoring system}

The subject's responses were in five points Likert scale ranging from (5-1) $5=$ always, $4=$ often, $3=$ sometimes \& 1 = never. 


\section{Methods}

1. Official permission from the authoritative personnel to conduct the study were obtained before starting the study

2. Tools of the study was translated into Arabic and presented to nine experts from fields of Nursing Administration and Nursing Psychology to check content validity, based on the experts' responses and pilot testing the questionnaire was adjusted and finalized.

3. Pilot study was conducted on $10 \%$ from nursing managers who were excluded from the study population, to identify the obstacles and problems that may be encountered in data collection.

4. Reliability of the tools were tested using Cronbach coefficient Alpha test, tool (1) was .844, Tool (2) was.745, Tool (3) was .724 and tool (4) was .783.

5. The program time was 20 hours. Two sessions every day for five days, every session 2 hours. Data were collected by the researchers during March 2013

6. For data collection. At the beginning the researchers distributed the questionnaire and collected it after 30 minutes. The researchers were present during collection of data for any needed guidance and clarification. The questionnaire collected immediately after filled.

7. An educational program was designed and implemented by the researchers to enhance nursing managers' transformational leadership, conflict management styles and decision making effectiveness.

8. Nursing managers' knowledge were tested by (Tool1) pre and post program.

9. Nursing managers' leadership behavior, conflict style and decision taking were assessed by (tool $2,3 \& 4$ ) pre and post program.

10. Ethical consideration: all participants interviewed for explaining the purposes and procedures of the study, and they have the right to withdrawal from the study at any time of the study. Oral consent to participate was assumed of filling the questionnaire sheet.

\section{Field work:}

Construction of the program

The first step in constructing this program was the statement of the instructional objectives. These objectives were derived from the assessed need.

\section{Program objectives}

The main objective of the program is to enhance nursing managers' transformational leadership, conflict management styles and decision making effectiveness.

\section{Specific Objectives}

At the end of the program the nursing mangers should be able to

- Define transformational leadership, conflict management styles and decision making

- Identify components of transformational leadership

- Recognize problems and best ways to make an effective decision

- List attributes of transformational leadership and efficient decision maker

- $\quad$ Enumerate decision making process steps

- Differentiate between conflicts management styles

○ Apply decision making steps / process to nursing manager work practice

\section{Selection and organization of program content}

After determining the objectives of the program, the content was specifically designed, methods of teaching and evaluation was identified. The content was selected after careful assessment of nursing managers ' needs. The use of simple scientific and professional language was considered

\section{Selection of the teaching method}

This is the third step in constructing the program. The selection of teaching methods was carried out according to the subjects and the educational principle.

The methods used were lecture, discussion and, visual aid.

\section{Evaluation of the program}

Evaluation of the effectiveness of the program is the final step that was planned to determine the extent to which they have acquired the knowledge and practiced it. To ensure that nursing managers' transformational leadership, conflict management styles and decision making were enhanced. 
Evaluation of nursing managers prior to the program was done in the form of pretest administered to them during the assessment of needs. At the end of the program, immediate post test was carried out for only 20 nursing managers. The same pre test used for immediate post test of nursing manager

\section{Statistical analysis:}

The collected data was organized, tabulated, and statistically analyzed using Microsoft Excel and Statistical package for the social sciences (SPSS), version 16. For quantitative data the range, mean, and standard deviation were used. The difference between two means was statistically analyzed using the student paired (t) test. For qualitative data the number and percent distribution was calculated. Chi square was used as a test of significant. Significant was at $\mathrm{p}<0.05$ for interpretation of results of tests of significant. Spearman's coefficient correlation test was used to show variables correlation. Correlation is significant at the 0.01 level

\section{Results}

Table (1): Shows characteristics of nurse managers including age, years of experience, administrative years of experience and position. It was observed that fifty (50.00\%) of nursing managers were in the age group $36-45$ years and equal percent $(25.0 \%)$ of them were in age group 25-35 \& 41-<50 years. Mean age 40.20 \pm 6.902 and range 26 years. More than fifty (55.00\%) nursing managers had 10-20 years of experience, $30.00 \%$ of them had more than 20 years and $15 \%$ had $<10$ years of experience. Mean years of experience $16.60 \pm 6.52$ with range 27 years. Regarding administrative years of experience, more than fifty $(55.00 \%)$ of nursing managers had more than 10 years of experience, $30.00 \%$ of them had $<10$ years and $15 \%$ had 10 years of experience. Mean years of administrative experience $11.80 \pm 5.14$ and range 17.00 years. Majority $(85.0 \%)$ of nursing managers were in head nurse position and only $15.0 \%$ of them under secretary nursing.

Table (2): Represents levels of nursing managers' knowledge about transformational leadership, conflict management, and decision making pre \& post the educational program. It was observed that levels of nursing managers' total knowledge and all items of transformational leadership, conflict management, and decision making were significant improved post than pre program at $p \leq 0.05$. All nursing managers' levels of total knowledge were poor at pre program and decreased to $5.0 \%$ at post program. In specific, none of nursing manager had good level of transformational leadership, conflict management, and decision making knowledge respectively at pre program, which improved to reach $90 \%, 85 \% \& 70 \%$ respectively at post program.

Table (3): Displays difference of nursing manager transformational leadership skills pre and post program. The table shows statistically significant improvement of nursing managers mean score in all items of transformational leadership skills post than pre program at $\mathrm{p} \leq 0.05$. Nursing managers pre program mean scores were $(3.385 \pm 0.987,3.156 \pm 1.008,2.825 \pm 1.271 \& 2.771 \pm 1.158)$ respectively significantly increased post program $(4.742 \pm 0.451,4.437 \pm 0.751,4.458 \pm 0.821, \& 4.60 \pm 0.542)$ respectively in individualized consideration, idealized influence, intellectual stimulation and inspirational motivation respectively.

Table (4): Describes distribution of nursing managers according their uses of conflict management styles pre and post program. As noticed in the table there was significant improvement at nursing managers levels in using of conflict management styles post program than pre program at $\mathrm{p} \leq 0.05$. Pre program, low and equal percent $(15 \% \& 10 \%)$ of nursing managers were strong in using of competition, compromise, avoidance and accommodation styles. As well as, $20 \%$ of them were strong in using of collaboration strategy, which changed post program and reached to all nursing manager were strong in using competition style, and highest percent $(95 \%, 90 \% \& 85 \%)$ of them were strong in using of compromise, avoidance, collaboration and accommodation styles respectively.

Table (5): Represents difference of nursing managers' factors influencing decision making pre \& post program. As noticed in the table there was significant differences in all factors influencing nursing managers' decision making in pre than post except achieving the revenue and profit and, health insurance factors. Pre program, considerable percent $(60,35,25, \& 20)$ of nursing managers were rarely and never considered administrative experience, regulations and laws, educational level or job satisfaction influencing their decisions, but post program all of them were always and often considered that these items influencing their decisions.

Table (6): Presents difference of nursing managers' best ways to make administrative decisions pre and post program. It was observed that there was significant improvement of nursing managers' ways to make administrative decisions post program than pre program at $\mathrm{p} \leq 0.05$. Pre program, equal percent $(70 \%)$ of nursing managers rarely and never relies on psychological and personal factors, away from risk or bear responsibility and, arbitrariness and lobbying ways when they making a decision, as well as equal percent (65\%) 
of them relies on personal experience and, laws and regulations, these percent was improved $(p \leq 0.05)$ post program as evidenced by all of them always and often relies on counseling with employers of previous administrative experience, laws and regulations, and majority (equal percent $95 \%$ and $85 \%$ ) always and often convene a meeting of the decision-making, preservation of human relations in the organization in the first place, and rely on personal experience, respectively.

Table (7): Represents difference of nursing managers' reported problems that affect the managerial decision-making pre and post program. The table shows statistically significant improvement of nursing managers managers' reported problems that affect the managerial decision-making post program than pre program at $\mathrm{p} \leq 0.05$. The table implied that highest percent of nursing managers ranged from $50 \%$ to $100 \%$ always and often reported that all these problems affect the managerial decision-making pre program, but these percent decreased to $25 \%$ to $50 \%$ post program.

Table (8): Represents difference of nursing managers' effectiveness of decision-making pre and post program. The table implied that statistically significant improvement of nursing managers' effectiveness of decision-making post program than pre program at $\mathrm{p} \leq 0.05$. Pre program, lowest percent (ranged from $10 \%$ $50 \%$ ) of nursing managers always and often makes an effective decision these percents were changed post program to almost all nursing managers always and often make effective decision in all items except employees face no major obstacles in the implementation of the decisions taken, and when making their decisions, they chose the easier alternative for the practical application items that reached to $85 \% \& 75 \%$.

Table (9) Reveals correlation between conflict management style, decision making effectiveness, transformational leadership and total level of knowledge post the educational program. Significant positive correlation at $\mathrm{p} \leq 0.05$ was found between nursing managers' total level of knowledge and conflict management styles, decision making effectiveness as well as four dimensions of transformational leadership.

Table (1): Nursing managers' characteristics

\begin{tabular}{|c|c|c|}
\hline Characteristics & No $(\mathrm{N}=20)$ & $\%$ \\
\hline $\begin{array}{ll}\text { Age } & \\
\bullet & 25-35 \\
\end{array}$ & 5 & $25.0 \%$ \\
\hline $36-45$ & 10 & $50.0 \%$ \\
\hline $\begin{array}{ll} & 41-50 \\
\end{array}$ & 5 & $25.0 \%$ \\
\hline $\begin{array}{l}\text { Mean } \pm \text { SD } \\
\text { Range }\end{array}$ & & $\begin{array}{l}1 \pm 6.902 \\
26\end{array}$ \\
\hline \multicolumn{3}{|l|}{ Years of experience } \\
\hline - $\quad<10$ years & 3 & $15.0 \%$ \\
\hline $10-20$ & 11 & $55.0 \%$ \\
\hline More than 20 & 6 & $30 . \%$ \\
\hline $\begin{array}{l}\text { Mean } \pm \text { SD } \\
\text { Range }\end{array}$ & & $\begin{array}{l}0 \pm 6.52 \\
27\end{array}$ \\
\hline \multicolumn{3}{|l|}{ Administrative years of experience } \\
\hline$\bullet \quad<10$ years & 6 & $30.0 \%$ \\
\hline 10 & 3 & $15.0 \%$ \\
\hline - $\quad$ More than 10 & 11 & $55.0 \%$ \\
\hline $\begin{array}{l}\text { Mean } \pm \text { SD } \\
\text { Range }\end{array}$ & & $\begin{array}{l}0 \pm 5.14 \\
7.00\end{array}$ \\
\hline \multicolumn{3}{|l|}{ Position } \\
\hline $\begin{array}{ll}- & \text { Under Secretary Nursing } \\
- & \text { Nurse manager }\end{array}$ & $\begin{array}{c}3 \\
17\end{array}$ & $\begin{array}{l}15.0 \% \\
85.0 \%\end{array}$ \\
\hline
\end{tabular}

Table (2): Levels of nursing managers' knowledge about $\backslash$ transformational leadership conflict management style and decision making pre \& post I program

\begin{tabular}{|c|c|c|c|c|c|c|c|}
\hline Dimensions & \multicolumn{3}{|c|}{ Pre $(\mathrm{N}=20)$} & \multicolumn{3}{|c|}{ Post $(\mathrm{N}=20)$} & \multirow{3}{*}{$\begin{array}{r}\mathbf{X}^{2} \\
\mathbf{P}\end{array}$} \\
\hline & \multirow{2}{*}{$\begin{array}{l}\text { Poor } \\
\mathbf{N} \\
\% \\
\end{array}$} & \multirow{2}{*}{$\begin{array}{l}\text { Fair } \\
\mathbf{N} \\
\% \\
\end{array}$} & \multirow{2}{*}{$\begin{array}{l}\text { Good } \\
\mathbf{N} \\
\% \\
\end{array}$} & \multirow{2}{*}{$\begin{array}{l}\text { Poor } \\
\mathbf{N} \\
\% \\
\end{array}$} & \multirow{2}{*}{$\begin{array}{l}\text { Fair } \\
\mathbf{N} \\
\% \\
\end{array}$} & Good & \\
\hline & & & & & & $\mathbf{N} \%$ & \\
\hline Transformational leadership & $\begin{array}{l}18 \\
90\end{array}$ & $\begin{array}{l}2 \\
10\end{array}$ & $\begin{array}{l}0 \\
00\end{array}$ & $\begin{array}{l}0 \\
00\end{array}$ & $\begin{array}{l}3 \\
15\end{array}$ & $\begin{array}{l}17 \\
85\end{array}$ & $\begin{array}{l}35.200 \\
0.000 *\end{array}$ \\
\hline Conflict management & $\begin{array}{l}17 \\
85\end{array}$ & $\begin{array}{l}3 \\
15\end{array}$ & $\begin{array}{l}0 \\
00\end{array}$ & $\begin{array}{l}1 \\
5\end{array}$ & $\begin{array}{l}1 \\
5\end{array}$ & $\begin{array}{l}18 \\
90\end{array}$ & $\begin{array}{l}33.44 \\
0.000^{*}\end{array}$ \\
\hline Decision making & $\begin{array}{l}19 \\
95\end{array}$ & $\begin{array}{l}1 \\
5\end{array}$ & $\begin{array}{l}0 \\
00\end{array}$ & $\begin{array}{l}3 \\
15\end{array}$ & $\begin{array}{l}3 \\
15\end{array}$ & $\begin{array}{l}14 \\
70\end{array}$ & $\begin{array}{l}37.33 \\
0.000^{*}\end{array}$ \\
\hline Total & $\begin{array}{l}20 \\
100\end{array}$ & $\begin{array}{l}0 \\
00\end{array}$ & $\begin{array}{l}0 \\
00\end{array}$ & $\begin{array}{l}1 \\
5\end{array}$ & $\begin{array}{l}1 \\
5\end{array}$ & $\begin{array}{l}18 \\
90\end{array}$ & $\begin{array}{l}40.00 \\
0.005^{*}\end{array}$ \\
\hline
\end{tabular}


An Educational Program about Nursing Managers`Transformational Leadership....

Table (3): Difference of nursing manager transformational leadership skills pre and post program

\begin{tabular}{|c|c|c|c|c|c|c|}
\hline \multirow{2}{*}{ Transformational leadership skills } & \multicolumn{2}{|c|}{ Pre $(\mathrm{N}=20)$} & \multicolumn{2}{|c|}{ Post $(\mathrm{N}=20)$} & \multicolumn{2}{|c|}{ Paired $\mathbf{t}$} \\
\hline & Mean & SD & Mean & SD & $\mathbf{t}$ & p \\
\hline Idealized Influence & 3.156 & 1.008 & 4.437 & 0.751 & 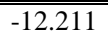 & $0.000 *$ \\
\hline Inspirational Motivation & 2.771 & 1.158 & 4.60 & 0.542 & -14.773 & $0.000^{*}$ \\
\hline Individualized Consideration & 3.385 & 0.987 & 4.742 & 0.451 & -12.514 & $0.000^{*}$ \\
\hline Intellectual Stimulation & 2.825 & 1.271 & 4.458 & 0.821 & -9.444 & $0.000^{*}$ \\
\hline
\end{tabular}

Table (4): Distribution of nursing managers according to their uses of conflict management styles pre and post program.

\begin{tabular}{|c|c|c|c|c|c|c|c|c|}
\hline \multirow{3}{*}{ Conflict styles } & \multicolumn{3}{|c|}{ Pre $(\mathrm{N}=20)$} & \multicolumn{4}{|c|}{ Post $(\mathrm{N}=20)$} & \multirow{3}{*}{$\begin{array}{l}\mathbf{X}^{2} \\
\mathbf{P}\end{array}$} \\
\hline & Strong & \multirow{2}{*}{$\begin{array}{l}\text { Average } \\
\mathbf{N} \\
\%\end{array}$} & \multirow{2}{*}{$\begin{array}{l}\text { Weak } \\
\mathbf{N} \\
\% \\
\end{array}$} & Strong & \multicolumn{2}{|c|}{ Average } & Weak & \\
\hline & $\mathbf{N} \quad \%$ & & & $\mathbf{N} \quad \%$ & $\begin{array}{l}\mathbf{N} \\
\% \\
\end{array}$ & & $\begin{array}{l}\mathbf{N} \\
\%\end{array}$ & \\
\hline Avoidance & $\begin{array}{l}2 \\
10 \% \\
\end{array}$ & $\begin{array}{l}9 \\
45 \% \\
\end{array}$ & $\begin{array}{l}9 \\
45 \% \\
\end{array}$ & $\begin{array}{l}19 \\
95 \% \\
\end{array}$ & $\begin{array}{l}1 \\
5 \% \\
\end{array}$ & & $\begin{array}{l}0 \\
00 \% \\
\end{array}$ & $\begin{array}{l}35.333 \\
0.004 * \\
\end{array}$ \\
\hline Competition & $\begin{array}{l}3 \\
15 \%\end{array}$ & $\begin{array}{l}9 \\
45 \%\end{array}$ & $\begin{array}{l}8 \\
40 \%\end{array}$ & $\begin{array}{l}20 \\
100 \%\end{array}$ & 0 & $00 \%$ & $\begin{array}{l}0 \\
00 \%\end{array}$ & $\begin{array}{l}37.333 \\
0.001 *\end{array}$ \\
\hline Compromise & $\begin{array}{l}3 \\
15 \% \\
\end{array}$ & $\begin{array}{l}13 \\
60 \% \\
\end{array}$ & $\begin{array}{l}4 \\
20 \% \\
\end{array}$ & $\begin{array}{l}19 \\
95 \% \\
\end{array}$ & $\begin{array}{l}1 \\
5 \% \\
\end{array}$ & & $\begin{array}{l}0 \\
00 \%\end{array}$ & $\begin{array}{l}38.000 \\
0.001 *\end{array}$ \\
\hline Accommodation & $\begin{array}{l}2 \\
10 \% \\
\end{array}$ & $\begin{array}{l}14 \\
70 \% \\
\end{array}$ & $\begin{array}{l}4 \\
20 \% \\
\end{array}$ & $\begin{array}{l}17 \\
85 \% \\
\end{array}$ & 3 & $15 \%$ & $\begin{array}{l}0 \\
00 \% \\
\end{array}$ & $\begin{array}{l}37.333 \\
0.000 * \\
\end{array}$ \\
\hline Collaboration & $\begin{array}{l}4 \\
20 \% \\
\end{array}$ & $\begin{array}{l}7 \\
35 \% \\
\end{array}$ & $\begin{array}{l}9 \\
45 \% \\
\end{array}$ & $\begin{array}{l}18 \\
90 \% \\
\end{array}$ & 2 & $10 \%$ & $\begin{array}{l}0 \\
00 \% \\
\end{array}$ & $\begin{array}{l}32.667 \\
.0005^{*}\end{array}$ \\
\hline
\end{tabular}

$* \mathrm{p} \leq 0.05$

Table (5): Difference of nursing managers' factors influencing decision making pre \& post program

\begin{tabular}{|c|c|c|c|c|c|c|c|}
\hline \multirow{3}{*}{ Factors } & \multicolumn{3}{|c|}{ Pre } & \multicolumn{3}{|c|}{ Post } & \multirow{3}{*}{$\begin{array}{l}\mathrm{X}^{2} \\
\mathrm{p}\end{array}$} \\
\hline & $\begin{array}{l}\text { Always } \\
\text { \&Often }\end{array}$ & $\begin{array}{l}\text { Some } \\
\text { times }\end{array}$ & $\begin{array}{l}\text { Rarely } \\
\text { \&Never }\end{array}$ & $\begin{array}{l}\text { Always } \\
\text { \&Often }\end{array}$ & $\begin{array}{l}\text { Some } \\
\text { times }\end{array}$ & $\begin{array}{l}\text { Rarely } \\
\text { \&Never }\end{array}$ & \\
\hline & $\mathbf{N} \%$ & $\mathbf{N} \%$ & $\mathbf{N} \%$ & $\mathbf{N} \%$ & $\mathbf{N} \%$ & $\mathbf{N} \%$ & \\
\hline 1.Administrative experience & $\begin{array}{l}6 \\
30 \%\end{array}$ & $\begin{array}{l}2 \\
10 \%\end{array}$ & $\begin{array}{l}12 \\
60 \%\end{array}$ & $\begin{array}{l}20 \\
100\end{array}$ & $\begin{array}{l}0 \\
00\end{array}$ & $\begin{array}{l}0 \\
00\end{array}$ & $\begin{array}{l}14.16 \\
0.005\end{array}$ \\
\hline 2. Medical specialty & $\begin{array}{l}8 \\
40 \%\end{array}$ & $5 \%$ & $\begin{array}{l}11 \\
55 \%\end{array}$ & $\begin{array}{l}12 \\
60\end{array}$ & $\begin{array}{l}8 \\
40\end{array}$ & $\begin{array}{l}0 \\
00\end{array}$ & $\begin{array}{l}17.44 \\
0.002 *\end{array}$ \\
\hline 3. Nationality & $\begin{array}{l}3 \\
15 \%\end{array}$ & $\begin{array}{l}3 \\
15 \%\end{array}$ & $\begin{array}{l}14 \\
70 \%\end{array}$ & $\begin{array}{l}6 \\
30\end{array}$ & $\begin{array}{l}1 \\
5\end{array}$ & $\begin{array}{l}13 \\
65\end{array}$ & $\begin{array}{l}12.85 \\
0.012\end{array}$ \\
\hline 4. Age & $\begin{array}{l}5 \\
25 \%\end{array}$ & $\begin{array}{l}0 \\
00 \%\end{array}$ & $\begin{array}{l}15 \\
75 \%\end{array}$ & $\begin{array}{l}17 \\
85\end{array}$ & $\begin{array}{l}2 \\
10\end{array}$ & $\begin{array}{l}1 \\
5\end{array}$ & $\begin{array}{l}21.600 \\
0.000 \text { * }\end{array}$ \\
\hline 5. Educational level & $\begin{array}{l}10 \\
50 \%\end{array}$ & $\begin{array}{l}5 \\
25 \%\end{array}$ & $\begin{array}{l}5 \\
25 \%\end{array}$ & $\begin{array}{l}20 \\
100\end{array}$ & $\begin{array}{l}0 \\
00\end{array}$ & $\begin{array}{l}0 \\
00\end{array}$ & $\begin{array}{l}13.40 \\
0.009 *\end{array}$ \\
\hline 6. Job satisfaction & $\begin{array}{l}9 \\
45 \% \\
\end{array}$ & $\begin{array}{l}7 \\
35 \% \\
\end{array}$ & $\begin{array}{l}4 \\
20 \% \\
\end{array}$ & $\begin{array}{l}20 \\
100 \\
\end{array}$ & $\begin{array}{l}0 \\
00 \\
\end{array}$ & $\begin{array}{l}0 \\
00 \\
\end{array}$ & $\begin{array}{l}18.31 \\
0.001 *\end{array}$ \\
\hline $\begin{array}{l}\text { 7. Achieving the revenue and } \\
\text { profit }\end{array}$ & $\begin{array}{l}14 \\
70 \%\end{array}$ & $\begin{array}{l}1 \\
5 \%\end{array}$ & $\begin{array}{l}5 \\
25 \%\end{array}$ & $\begin{array}{l}19 \\
95\end{array}$ & $\begin{array}{l}1 \\
5\end{array}$ & $\begin{array}{l}0 \\
00\end{array}$ & $\begin{array}{l}7.30 \\
0.120\end{array}$ \\
\hline 8. Regulations, and laws & $\begin{array}{l}9 \\
45 \%\end{array}$ & $\begin{array}{l}4 \\
20 \%\end{array}$ & $\begin{array}{l}7 \\
35 \%\end{array}$ & $\begin{array}{l}20 \\
100\end{array}$ & $\begin{array}{l}0 \\
00\end{array}$ & $\begin{array}{l}0 \\
00\end{array}$ & $\begin{array}{l}17.68 \\
0.001 \text { * }\end{array}$ \\
\hline 9. Environmental factors & $\begin{array}{l}4 \\
20 \%\end{array}$ & $\begin{array}{l}4 \\
20 \%\end{array}$ & $\begin{array}{l}12 \\
60 \%\end{array}$ & $\begin{array}{l}18 \\
90\end{array}$ & $\begin{array}{l}1 \\
5\end{array}$ & $\begin{array}{l}1 \\
5\end{array}$ & $\begin{array}{l}22.781 \\
0.000 *\end{array}$ \\
\hline 10. Health insurance & $\begin{array}{l}10 \\
50 \%\end{array}$ & $\begin{array}{l}2 \\
10 \%\end{array}$ & $\begin{array}{l}8 \\
40 \%\end{array}$ & $\begin{array}{l}13 \\
65\end{array}$ & $\begin{array}{l}2 \\
10\end{array}$ & $\begin{array}{l}5 \\
25\end{array}$ & $\begin{array}{l}1.339 \\
0.855\end{array}$ \\
\hline
\end{tabular}


An Educational Program about Nursing Managers` Transformational Leadership....

Table (6): Difference of nursing managers' best ways to make administrative decisions pre and post program.

\begin{tabular}{|c|c|c|c|c|c|c|c|}
\hline \multirow{3}{*}{ Items } & \multicolumn{3}{|c|}{ Pre } & \multicolumn{3}{|c|}{ Post } & \multirow{3}{*}{$\begin{array}{l}\mathbf{X}^{2} \\
\mathbf{p}\end{array}$} \\
\hline & $\begin{array}{l}\text { Always } \\
\text { \&Often }\end{array}$ & \multirow{2}{*}{$\begin{array}{r}\begin{array}{l}\text { Some } \\
\text { times }\end{array} \\
\mathbf{N} \% \\
\end{array}$} & $\begin{array}{l}\text { Rarely\& } \\
\text { Never }\end{array}$ & $\begin{array}{l}\text { Always } \\
\text { \& Often }\end{array}$ & $\begin{array}{l}\text { Some } \\
\text { times }\end{array}$ & $\begin{array}{l}\text { Rarely\& } \\
\text { Never }\end{array}$ & \\
\hline & $\mathrm{N} \%$ & & N \% & N \% & N \% & $\mathbf{N} \%$ & \\
\hline $\begin{array}{l}\text { 2. Counseling with employers of } \\
\text { previous administrative experience }\end{array}$ & $\begin{array}{c}9 \\
45\end{array}$ & $\begin{array}{l}1 \\
5\end{array}$ & $\begin{array}{l}10 \\
50\end{array}$ & $\begin{array}{c}20 \\
100\end{array}$ & $\begin{array}{c}0 \\
00\end{array}$ & $\begin{array}{c}0 \\
00\end{array}$ & $\begin{array}{l}22.707 \\
0.000 *\end{array}$ \\
\hline $\begin{array}{l}4 . \quad \text { Taking into account laws and } \\
\text { regulations }\end{array}$ & $\begin{array}{c}5 \\
25 \\
\end{array}$ & $\begin{array}{c}2 \\
10\end{array}$ & $\begin{array}{l}13 \\
65\end{array}$ & $\begin{array}{c}20 \\
100\end{array}$ & $\begin{array}{c}0 \\
00 \\
\end{array}$ & $\begin{array}{c}0 \\
00 \\
\end{array}$ & $\begin{array}{l}25.714 \\
0.000 *\end{array}$ \\
\hline $\begin{array}{l}5 . \\
\text { place }\end{array} \quad$ Making a profit in the first & $\begin{array}{c}3 \\
15\end{array}$ & $\begin{array}{c}5 \\
25\end{array}$ & $\begin{array}{l}12 \\
60\end{array}$ & $\begin{array}{l}12 \\
60\end{array}$ & $\begin{array}{c}3 \\
15\end{array}$ & $\begin{array}{c}5 \\
25\end{array}$ & $\begin{array}{c}9.143 \\
0.058^{*}\end{array}$ \\
\hline $\begin{array}{l}6 . \quad \text { Taking into account along } \\
\text { with marketing and advertising }\end{array}$ & $\begin{array}{c}5 \\
25\end{array}$ & $\begin{array}{c}3 \\
15\end{array}$ & $\begin{array}{l}12 \\
60\end{array}$ & $\begin{array}{c}9 \\
45\end{array}$ & $\begin{array}{c}8 \\
40\end{array}$ & $\begin{array}{c}3 \\
15\end{array}$ & $\begin{array}{l}15.958 \\
0.003 *\end{array}$ \\
\hline $\begin{array}{l}9 . \quad \text { Preservation of human } \\
\text { relations in the organization in the first } \\
\text { place }\end{array}$ & $\begin{array}{c}5 \\
25\end{array}$ & $\begin{array}{c}4 \\
20\end{array}$ & $\begin{array}{l}11 \\
55\end{array}$ & $\begin{array}{l}19 \\
95\end{array}$ & $\begin{array}{c}0 \\
00\end{array}$ & $\begin{array}{c}0 \\
00\end{array}$ & $\begin{array}{l}28.000 \\
0.000 *\end{array}$ \\
\hline Arbitrariness and lobbying & $\begin{array}{c}2 \\
15\end{array}$ & $\begin{array}{c}4 \\
20\end{array}$ & $\begin{array}{l}14 \\
70\end{array}$ & $\begin{array}{c}9 \\
45\end{array}$ & $\begin{array}{c}4 \\
20\end{array}$ & $\begin{array}{c}7 \\
35\end{array}$ & $\begin{array}{l}16.586 \\
0.002 *\end{array}$ \\
\hline
\end{tabular}

$* p \leq 0.05$

Table (7): Difference of nursing managers' reported problems that affect the managerial decision-making pre and post program

\begin{tabular}{|c|c|c|c|c|c|c|c|}
\hline \multirow{3}{*}{ Problems } & \multicolumn{3}{|c|}{ Pre } & \multicolumn{3}{|c|}{ Post } & \multirow{3}{*}{$\begin{array}{l}\mathrm{X}^{2} \\
\mathrm{P}\end{array}$} \\
\hline & \multirow{2}{*}{$\begin{array}{l}\text { Always } \\
\text { \&Often } \\
\text { N } \%\end{array}$} & $\begin{array}{l}\text { Some } \\
\text { times }\end{array}$ & $\begin{array}{l}\text { Rarely\& } \\
\text { Never }\end{array}$ & $\begin{array}{l}\text { Always } \\
\text { \& Often }\end{array}$ & $\begin{array}{l}\text { Some } \\
\text { times }\end{array}$ & $\begin{array}{l}\text { Rarely\& } \\
\text { Never }\end{array}$ & \\
\hline & & N $\%$ & $\mathrm{~N} \quad \%$ & $\mathrm{~N} \quad \%$ & $\mathbf{N} \%$ & $\mathbf{N} \%$ & \\
\hline \multirow[b]{2}{*}{ 1. Lack of job satisfaction } & 19 & 0 & 1 & 7 & 2 & 11 & 15.958 \\
\hline & $95 \%$ & $00 \%$ & $5 \%$ & $35 \%$ & $10 \%$ & $55 \%$ & 0.003 * \\
\hline \multirow{2}{*}{$\begin{array}{l}\text { 2. Human relations in the } \\
\text { organization of health }\end{array}$} & 19 & 1 & 0 & 6 & 5 & 9 & 18.917 \\
\hline & $95 \%$ & $5 \%$ & $00 \%$ & $30 \%$ & $25 \%$ & $45 \%$ & $0.001 *$ \\
\hline \multirow{2}{*}{ 3. Lack of appropriate services } & 19 & 1 & 0 & 8 & 10 & 2 & 21.253 \\
\hline & $95 \%$ & $5 \%$ & $00 \%$ & $40 \%$ & $50 \%$ & $10 \%$ & $0.000 *$ \\
\hline \multirow{2}{*}{ 4. The health insurance } & 10 & 2 & 8 & 5 & 7 & 8 & 6.111 \\
\hline & $50 \%$ & $10 \%$ & $40 \%$ & $25 \%$ & $35 \%$ & $40 \%$ & 0.191 \\
\hline \multirow{2}{*}{ 5. Work stress } & 19 & 1 & 0 & 7 & 3 & 10 & 27.222 \\
\hline & $95 \%$ & $5 \%$ & $00 \%$ & $35 \%$ & $15 \%$ & $50 \%$ & $0.000 *$ \\
\hline \multirow{2}{*}{ 6. Environmental factors } & 19 & 1 & 0 & 5 & 6 & 9 & 21.333 \\
\hline & $95 \%$ & $5 \%$ & $00 \%$ & $25 \%$ & $30 \%$ & $45 \%$ & $0.000 *$ \\
\hline \multirow{2}{*}{$\begin{array}{l}\text { 7. Lack of supervision and } \\
\text { inspection }\end{array}$} & 15 & 5 & 0 & 7 & 2 & 11 & 15.202 \\
\hline & $75 \%$ & $25 \%$ & $00 \%$ & $35 \%$ & $10 \%$ & $55 \%$ & 0.004 * \\
\hline \multirow{2}{*}{ 8. Weak managerial experience } & 19 & 1 & 0 & 10 & 0 & 10 & 15.638 \\
\hline & $95 \%$ & $5 \%$ & $00 \%$ & $50 \%$ & $00 \%$ & $50 \%$ & 0.001 * \\
\hline \multirow{2}{*}{ 9. Differences in specialty } & 19 & 1 & 0 & 6 & 4 & 10 & 21.271 \\
\hline & $95 \%$ & $5 \%$ & $00 \%$ & $30 \%$ & $20 \%$ & $50 \%$ & $0.000 *$ \\
\hline \multirow{2}{*}{ 10. Regulations, and laws } & & 0 & 0 & 6 & 4 & 10 & 25.600 \\
\hline & $100 \%$ & $00 \%$ & $00 \%$ & $30 \%$ & $20 \%$ & $50 \%$ & $0.000 *$ \\
\hline
\end{tabular}

${ }^{*} \mathrm{p} \leq 0.05$ 
Table (8): Difference of nursing managers' effectiveness of decision-making pre and post

\begin{tabular}{|c|c|c|c|c|c|c|c|}
\hline \multirow{3}{*}{ Items } & \multicolumn{3}{|c|}{ Pre } & \multicolumn{3}{|c|}{ Post } & \multirow{3}{*}{$\begin{array}{c}\mathrm{X}^{2} \\
\mathrm{p}\end{array}$} \\
\hline & $\begin{array}{l}\text { Always } \\
\text { \&Often }\end{array}$ & $\begin{array}{l}\text { Some } \\
\text { times }\end{array}$ & $\begin{array}{l}\text { Rarely\& } \\
\text { Never }\end{array}$ & $\begin{array}{l}\text { Always } \\
\text { \& Often }\end{array}$ & $\begin{array}{l}\text { Some } \\
\text { times }\end{array}$ & $\begin{array}{l}\text { Rarely\& } \\
\text { Never }\end{array}$ & \\
\hline & N $\%$ & N \% & N \% & $\mathrm{N} \%$ & N $\%$ & N \% & \\
\hline \multirow{2}{*}{$\begin{array}{l}\text { 1. I took my decisions in line with the } \\
\text { objectives of the organization }\end{array}$} & 5 & 4 & 11 & 20 & 0 & 0 & 26.842 \\
\hline & 25 & 20 & 55 & 100 & 00 & 00 & $0.000 *$ \\
\hline \multirow{2}{*}{$\begin{array}{l}\text { 2. I believe that my decisions are } \\
\text { meeting its goals }\end{array}$} & 2 & 8 & 10 & 20 & 0 & 0 & 33.333 \\
\hline & 10 & 40 & 50 & 100 & 00 & 00 & $0.000 *$ \\
\hline \multirow{2}{*}{$\begin{array}{l}\text { 3. When making my decisions, I } \\
\text { chose the easier alternative for the } \\
\text { practical application }\end{array}$} & 4 & 2 & 14 & 15 & 4 & 1 & 20.238 \\
\hline & 20 & 10 & 70 & 75 & 20 & 5 & $0.000 *$ \\
\hline \multirow{2}{*}{$\begin{array}{l}\text { 4. Employees face no major obstacles } \\
\text { in the implementation of the } \\
\text { decisions taken }\end{array}$} & 7 & 2 & 11 & 17 & 3 & 0 & 23.533 \\
\hline & 35 & 10 & $\begin{array}{l}11 \\
55\end{array}$ & 85 & 15 & 00 & $0.000 *$ \\
\hline \multirow{3}{*}{$\begin{array}{l}\text { 5. I take into account the capacity } \\
\text { and knowledge of the employees } \\
\text { that necessary to implement the } \\
\text { decisions taken }\end{array}$} & & & & & & & \\
\hline & 10 & 0 & 10 & 20 & 0 & 0 & 18.560 \\
\hline & 50 & 00 & 50 & 100 & 00 & 00 & $0.000 *$ \\
\hline \multirow{2}{*}{$\begin{array}{l}\text { 6. I have taken my decisions at the } \\
\text { appropriate time }\end{array}$} & 10 & 0 & 10 & 20 & 0 & 0 & 18.333 \\
\hline & 50 & 00 & 50 & 100 & 00 & 00 & $0.000 *$ \\
\hline \multirow{2}{*}{$\begin{array}{l}\text { 7. I had the ability to make quick } \\
\text { decisions to face problems of } \\
\text { emergency }\end{array}$} & 6 & 1 & 13 & 19 & 0 & 1 & 22.545 \\
\hline & 30 & 5 & 65 & 95 & 00 & 5 & $0.000 *$ \\
\hline \multirow{2}{*}{$\begin{array}{l}\text { 8. Decisions that I have taken by the } \\
\text { acceptance of concerned employees } \\
\text { is implemented }\end{array}$} & 6 & 4 & 10 & 19 & 2 & 0 & 17.067 \\
\hline & 30 & 20 & 50 & 95 & 10 & 00 & $0.002 *$ \\
\hline \multirow{2}{*}{$\begin{array}{l}\text { 9. I consulted with employees when } \\
\text { making decisions to facilitate the } \\
\text { acceptance of these decisions }\end{array}$} & 7 & 3 & 10 & 20 & 0 & 0 & 25341 \\
\hline & 35 & 15 & 50 & 100 & 00 & 00 & $0.000 *$ \\
\hline
\end{tabular}
*p $\leq 0.05$

Table (9): Correlation between transformational, leadership conflict management style, decision making effectiveness, and total level of knowledge post the educational program

\begin{tabular}{|c|c|c|c|c|c|c|c|c|}
\hline \multicolumn{2}{|l|}{ Variables } & 1 & 2 & 3 & 4 & 5 & 6 & 7 \\
\hline \multirow{2}{*}{ 1.Idealized Influence } & $\mathrm{r}$ & 0.178 & 0.423 & 1.000 & 0.556 & 0.356 & 0.117 & 0.671 \\
\hline & $P$ & 0.226 & $0.003 *$ & $\ldots$ & $0.005^{* * *}$ & 0.062 & 0.312 & $0.002^{*}$ \\
\hline \multirow{2}{*}{$\begin{array}{r}\text { 2.Inspirational } \\
\text { Motivation }\end{array}$} & $\mathrm{r}$ & 0.321 & 0.590 & 0.556 & 1.000 & 0.454 & 0.115 & 0.557 \\
\hline & $P$ & 0.084 & $0.007^{*}$ & $0.005^{* * *}$ & -- & $0.022^{*}$ & 0.314 & $0.004 *$ \\
\hline \multirow{2}{*}{$\begin{array}{l}\text { 3.Individualized } \\
\text { Consideration }\end{array}$} & $\mathrm{r}$ & 0.068 & 0.490 & 0.356 & 0.454 & 1.000 & 0.454 & -0.682 \\
\hline & $P$ & 0.387 & $0.028 *$ & 0.062 & $0.022 *$ & -- & $0.002 *$ & $0.003^{*}$ \\
\hline \multirow{2}{*}{ 4.Intellectual Stimulation } & $\mathrm{r}$ & 0.447 & 0.418 & 0.117 & 0.115 & 0.454 & 1.000 & 0.577 \\
\hline & $P$ & $0.024^{*}$ & $0.033^{*}$ & 0.312 & 0.314 & $0.002 *$ & -- & $0.009 *$ \\
\hline \multirow{2}{*}{$\begin{array}{c}5 . \text { Conflict management } \\
\text { styles }\end{array}$} & $\mathrm{r}$ & 1.000 & 0.659 & 0.178 & 0.321 & 0.068 & 0.447 & 1.000 \\
\hline & $P$ &.-- & $0.001 * *$ & 0.226 & 0.084 & 0.387 & $0.024 *$ & \\
\hline \multirow{2}{*}{$\begin{array}{cc}\begin{array}{c}\text { 6.Decision } \\
\text { effectiveness }\end{array} & \text { making }\end{array}$} & $\mathrm{r}$ & 0.659 & 1.000 & 0.423 & 0.590 & 0.490 & 0.418 & 0.960 \\
\hline & $P$ & $0.001 * *$ & -- & $0.003 *$ & $0.007^{*}$ & $0.028^{*}$ & $0.033^{*}$ & $0.000^{*}$ \\
\hline \multirow{2}{*}{$\begin{array}{l}\text { 7.Total level of } \\
\text { knowledge }\end{array}$} & $\mathrm{r}$ & 1.000 & 0.960 & 0.671 & 0.557 & -0.682 & 0.577 & 0.645 \\
\hline & $P$ & & $0.000 *$ & $0.002 *$ & $0.004^{*}$ & $0.003 *$ & $0.009 *$ & $0.003 *$ \\
\hline
\end{tabular}

** Correlation is significant at the 0.01 level.

\section{Discussion}

Leadership development, "leadership training" or "management training" have been used interchangeably in both scientific and practitioner literature. All of them serve the purpose of supporting individuals to perform effectively in managerial roles. The importance of training in organizations has increased over the past decades. Rapid changes in the environment of organizations such as the global economy, the 
increasing pace of technological development and fierce competitions have produced new challenges for leaders. Offering effective leadership trainings that help leaders to enhance their skills and adapt to changing environments is an important acumen for training professionals (Radstaak, 2008).

Present results revealed that nursing managers' levels of total knowledge and all items of transformational leadership, conflict management, and decision making were significant $(\mathrm{p} \leq 0.05)$ improved post than pre program. All nursing managers' levels of total knowledge were poor at pre program and decreased to minor percent at post program. In specific, none of nursing manager had good level of transformational leadership, conflict management, and decision making knowledge respectively at pre program, which improved post program, (Table, 2). This result indicated that the program provides reinforcement and information for nursing managers, as well as increases their understanding about their own leadership style, conflict management, and decision making. In fact, nurse leaders must be given education to effectively manage the challenges they will face on their units. Organizations that foster the transformational style of leading will have positive organizational outcomes and contribute to improved clinical practice with better patient outcomes (Tourangeau \& McGilton, 2004).

Now days, knowledge is considered to be most essential asset in all types of organizations, still significant importance has not been given to knowledge management practices (Danish et al., 2013). Likewise, Nguyen \& Mohamed (2011) suggested that transactional and transformational leaders are part of knowledge management training. According to Liu, et al., (2010) knowledge caliber depends upon the willingness of sharing knowledge and group distinctiveness. Transformational leadership style supports generating and disseminating knowledge at organizational level, (both individual and group). Also, Zori \& Morrison, (2009) pointed out that effective functioning in the management role requires knowledge and skills. Yet the literature suggests that many nurse managers receive little or insufficient education and support for the manager role. Cummings et al., (2008) found that higher levels of education and experience led to increased leadership effectiveness. Also, Byram, (2000) stated that learning the practice of transformational leading could improve the manager's ability to deliver effective leadership skills and achieve better outcomes.

Fitzpatrick, (2007) concluded that it was important to establish a base of knowledge among our participants. Brooks \& Anderson's (2004) supported the present study results and showed that ongoing leadership skill training for hospital nurse managers is needed. Moreover, the 1999 Nursing Task Force Report noted that nurses lack formal leadership education (Tourangeau, 2003). Leadership, whether through inherited characteristics or learned experiences, can be enhanced through continuing education and training, therefore given the lack of formal education, continuing education programs are necessary to enrich nursing leaders' skills and competencies (Wicker, 2008).

The current results showed that pre program, nursing manager's mean scores were significantly $(\mathrm{p} \leq 0.05)$ increased post program than pre program in individualized consideration, idealized influence, intellectual stimulation and inspirational motivation (Table, 3). These results indicated that the nurse managers were practicing behaviors consistent with a transformational style of leading. Along with the present study results Kelloway, et al., (2000) found that statistical significant changes in transformational leadership resulting from training.

As noticed in the present study results individualized consideration had better scores at pre and post program when compared to the other subscales followed by inspirational motivation, post program (Table, 3 ). This result indicated that more leadership behaviors are perceived to be practiced in individualized consideration than in any one of the other subgroups. The course competencies captured the nurse leaders' experiences related to a transformational style of leading, thus, increasing their success at using effective leadership skills.

Individualized influence occurs when leaders earn the trust and respect of their followers by doing the right thing rather than ensuring that the subordinates do things right. When the leaders focus on doing the right thing, which they usually do by using stories and symbols to communicate their vision and their message, they serve as role models (Modassir \& Singh, 2008). Similarly, Dionne, et al., (2004) posited that by means of individualized consideration, a leader addresses issues of competence, meaningfulness and impact with each team member, and encourages continued individual development. Furthermore, transformational leaders' inspiration motivation may arouse shared vision and goal, high team cohesion and interpersonal trust among group members, which should motivate them to engage more intensive interaction, cooperate more closely with each other (Zohar \& Tenne-Gazit, 2008).

Conflict in the workplace is unavoidable, especially in nursing. There can be numerous conflicts nurses are confronted with on a daily basis. The outcome depends on how the conflict is managed or resolved (Leary, 2011). Leaders who described themselves as more transformational used integrative conflict management styles and had followers with greater levels of satisfaction (Copley, 2008).

The current study results showed that there was significant $(p \leq 0.05)$ improvement at nursing managers` levels in using of conflict management styles post program than pre program (Table, 4). This result indicated that education and training has an effect on the nursing managers` managing conflicts. Anthony et al., 
(2005) mentioned that conflict resolution is a key role of managers and a skill which every nurse manager needs to posses to retain staff. Furthermore, Welford, (2002) reported that through informal learning such as journal clubs, reflective groups, in-service sessions and committees, and through formal educational programs, nurses can develop a repertoire of contemporary knowledge and skills to enhance their roles. The present results are in alignment with studies of Brinkert, (2011) \& Deans, (2004) simply revealed the positive correlation with the acquisition of skills and competencies as important means for handling conflict and violence management situations.

Present study results revealed that pre program, low percent of nursing managers were strong in using of competition, compromise, avoidance and accommodation styles, as well as using of collaboration style, which changed post program and reached to all nursing manager were strong in using of competition style and majority of them were strong in using of compromise, avoidance, collaboration and accommodation styles, (Table, 4). These results reflect that, in-service and continuing education programs for nurse managers should be continually implemented. Staff development programs, using role play, videotape playbacks, debriefing sessions and case management, would assist all of them to become aware of how they can contribute to the overall management strategies used for conflicts.

Healthcare professionals deal with conflict every day. Nurses have to spend $19 \%$ of their working time to resolve (Pavlakis et al., 2011). Organizational conflict must not necessarily be reduced, suppressed, or eliminated, but managed to enhance organizational effectiveness. The decisions that are made in the process of managing conflict must be ethical and should satisfy the needs and expectations of the relevant stakeholder (Rahim, 2002).

Rahim, (2002) mentioned that there is no one best style for dealing with different situations effectively. A style is considered appropriate for a conflict situation if its use leads to effective formulation and/or solution to a problem. Others have attempted to relate the various styles of handling interpersonal conflict of the organizational participants and their effects on quality of problem solution or attainment of social system objectives. It becomes evident that the distinction between the "amount of conflict" at various levels and the "styles of handling interpersonal conflict," is essential for a proper understanding of the nature of conflict management

A large proportion of the current respondents pre program and all of them post program chooses competition as a strategy to handle conflict. This may be due to their limited time for discussion and a prompt (unplanned) decision. This finding is contradictory with Kaitelidou et al., (2012); Pavlakis et al., (2011); Mahon \& Nicoreta, (2011) they found most participants chooses the avoidance strategy to managing conflict. While, Hendel, et al., (2005) averred that compromising was found to be the most frequent mode chosen in conflict management. And the least frequent mode was found to be accommodating.

Current study results revealed that there was a significant $(\mathrm{p} \leq 0.05)$ difference in all factors influencing nursing managers' decision making in pre than post except achieving the revenue and profit and, health insurance factors (Table, 5). The findings indicated that when nursing managers gain knowledge and skills in their work, they are able to employ this expertise to identify the major factors that affect decision making process with ease. They become successful in problem-solving and effective in decision-making. Moreover, educating nurse managers and team members about different decision-making procedures cultivates criticalthinking skills.

In consistent with the present study results Thompson et al., (2002) found that a greater recognition of the potential for teaching decision making skills and models in basic and continuing education and training is needed. On the other hand, Fitzpatrick, (2007) concluded that training exercises are still limited due to time, space, financial considerations, or physical limitations to conduct a large number of trials/runs in a given (usually short) period of time. One way of providing a decision maker with the opportunity to make many decisions is to introduce the use of tactical decision-making simulations in his regular training schedule.

Decision-making is a purposeful process whereby the manager or group selects the most suitable alternative for the solution of a problem, implements it and evaluates the consequences thereof. Numerous factors affecting individuals and groups in the decision making process (Niekerk, 2005).

Present study results revealed that pre program, considerable percent of nursing managers were rarely and never considered administrative experience, rules and laws, educational level and, job satisfaction influencing their decisions, but post program all of them were always and often considered that these items influencing their decisions (Table, 5). These results means that the provision of continuing education focused on enriching nurses' knowledge and skill related to factor affecting decision making process. This strategy is consistent with the College of Nurses of Ontario's (2010) principles that life long learning, based on the learner's needs, is required for competent practice.

Along line with the present study results Effken \& Verran (2010) concluded that nursing managerial experience correlated with effective decisions, but effective decision making was also influenced by motivation to manage and managerial style, as well as by the setting. Also, Dietrich (2010) stated that several factors 
influence decision making. These factors are including past experience, cognitive biases, age and individual differences, belief in personal relevance, and an escalation of commitment. Understanding the factors that influence decision making process is important to understanding what decisions are made. That is, the factors that influence the process may impact the outcomes.

Tanner (2006) in another study concluded that five principles factors influence decision making; internal factors includes variables such as decision maker's physical and emotional state, personal philosophy, interests, experience, knowledge, and risk taking and risk avoidance behavior. And external factors include environmental conditions, time, and resources. Similarly, a study by Gaskin, et al., (2012) on nursing managers, similar themes were found. The participants reported that personal issues, lack of consultation and influence to implement change, managing expectations of others, heavy workloads and patient acuity contributed to the challenges they experienced in making decision. These challenges indicated that nursing managers need sound management skills.

Present study results revealed that there was a significant $(\mathrm{p} \leq 0.05)$ improvement of nursing managers' ways to make administrative decisions post program than pre program (Table, 6). Decision making is considered one of the most important everyday jobs in management and selecting the type and method of decision making is considered to be one of the most important skills of a manager. Additionally, nurse managers and supervisors from various health care organizations make different decisions during the day (Ahmed \& Safadi, 2013). Further, Bord Altranais (2000) specifies that nurses must maintain and develop competencies through continuous education and professional development.

Current study results revealed that pre program, more than two third of nursing managers rarely and never relies on psychological and personal factors, away from risk or bear responsibility and, arbitrariness and lobbying ways when they taking a decision, as well as about two third of them relies on personal experience, and laws and regulations when making decision, these percentage was significantly $(\mathrm{p} \leq 0.05)$ changed post program as evidenced by all of them always and often relies on counseling with employers of previous administrative experience and, laws and regulations, and, majority always and often convene a meeting of the decision-making, preservation of human relations in the organization in the first place, and rely on personal experience, (Table, 6 ).

Management scholars now agree that there is no one best approaches to make decisions, to lead, and to motivate (Rahim, 2002). In this regard, Michel (2007) suggested that organizations need to understand decision making to delegate decision-making activities to employees with success. The present study results are in alignment with Lorber \& Skela Savic, (2011) conducted which showed that nursing leaders did not involve their employees in the decision-making process. Also, Niekerk, (2005) has shown that many decisions are taken without consultation or involvement of others.

Present study results revealed statistically significant $(\mathrm{p} \leq 0.05)$ improvement of nursing managers managers' reported problems that affect the managerial decision-making post program than pre program. Highest percent of nursing managers always and often reported that all these problems affect the managerial decision-making pre program, but these percent decreased post program (Table, 7). This may be due to that the nurse managers may not be transferring their knowledge to their own management practices. Effken, et al., (2010) stated that given the variety of decisions that nurse managers must make, it is unlikely that anyone is an expert on every problem.

Current study results revealed that statistically significant $(\mathrm{p} \leq 0.05)$ improvement of nursing managers' effectiveness of decision-making post program than pre program at (Table, 8). Ellis \& Hartley (2005) mentioned that nurses who consistently make effective decisions develop and maintain a current knowledge base through active participation in continuing education programs, on-going self-study, and thoughtful reflection on clinical experience. Cherie \& Gebrekidan (2005) concluded that managers should recognize these attributes and be sensitive to the factors that affect decision-making, change their method as appropriate, modify and mitigate detrimental influences when possible, and cope with those that cannot be changed. In this way, they will improve the effectiveness of decision-making.

Present study results revealed that lowest percent of nursing managers always and often makes an effective decision pre program, these percent was changed post program to almost all nursing managers always and often make effective decision in all items except employees face no major obstacles in the implementation of the decisions taken, and when making their decisions, they chose the easier alternative for the practical application items (Table, 8). This result may be due to the effect of education program. As leaders gain increased understanding of decision-making process, they may institute processes that better serve various levels of leadership. Nutt, (2006) posited that middle managers need education to understand the complexity of decisions at higher levels. In this regard, Benner, et al., (1996) reported that as nurses gain essential knowledge, thinking processes and experience, effective decision-making processes grow eventually. Ineffective decision making among nurses have different consequences on patients' outcomes especially in critical care units. 
Existing study results revealed significant positive correlation at $\mathrm{p} \leq 0.05$ was found between nursing managers' total level of knowledge and conflict management styles, decision making effectiveness as well as four dimensions of transformational leadership,(Table, 9). McGuire \& Kennerly, (2006) suggests that transformational leadership can increase nurse leader's ability to practice using an approach that includes others in decision-making. Transformational leading can be taught through courses, and encourage nurse leaders to change their practice to become a more engaged transformational type leader. Transformational leadership was found to affect significantly the conflict management strategy chosen (Hendel, et al., 2005). According to Pielstick,(1998) when leaders foster participation with others in the decision making process, there is less likelihood for escalating conflict to emerge. Moreover,_Cruz et al. (1999) admitted that directive leadership style had an important impact on the process of group information sampling. They proposed that leadership style impacted on the results of group decision-making, group members' perception of conflicts, self-confidence on decision-making and obedience to leaders

\section{Conclusion and recommendations}

Overall the evidence evaluating the effectiveness of nursing managers training programs for enhancing their transformational leadership, conflict management styles and, decision making effectiveness indicates that their knowledge and skills was generally poor pre program. An educational program was designed and implemented. After implementation of the program, their knowledge scores significantly improved and their skills positively changed.

\section{Based on the findings of this study, it is recommended that}

1. Early training, especially during undergraduate studies, is absolutely essential for an integrated knowledge of all aspects of leadership, decision making process and conflict and a constructive resolution process.

2. Hospitals administration and nurse managers' efforts must move in two different directions: a) take into account and remove all organizational factors that hinder decision making process or generate conflict and thus create a healthy work environment and culture that promotes effective decision making, collaboration and team work, b) initiate ongoing educational programs and training for the all nursing managers about role of transformational leader in taking effective decisions and resolution of conflicts.

3. Encourage inclusiveness by disseminating and translating research findings through activities such as presentations, consultation, educational programs, courses, activities, and journal clubs.

\section{References}

[1]. Roussel L \& Swansburg R, 2009. Management and leadership for nurses administrators, 5th edition, Jones and Bartlett, USA.

[2]. Robbins, S. P. and Coulter, M. (2007) Management (9th ed.). London: Prentice- Hall

[3]. Warrilow. S (2012) Transformational Leadership Theory - The 4 Key Components in Leading Change \& Managing Change. [Retrieved 15/03/2013]. http://EzineArticles.com/?expert=Stephen_Warrilow

[4]. Surakka T (2008) The nurse manager's work in the hospital environment during the 1990s and 2000s: responsibility, accountability and expertise in nursing leadership. J Nurs Manag 16(5):525-34

[5]. Bass, B. M., \& Avolio, B. J. (1999). Training full range leadership. Redwood City, CA: Mindgarden.

[6]. Hayati , Charkhabi M and Naami A. (2014). The relationship between transformational leadership and work engagement in governmental hospitals nurses: a survey study .(http://creativecommons.org/licenses/by/2.0)

[7]. Bass BM, Avolio BJ, Jung DI, Berson Y (2003) Predicting unit performance by assessing transformational and transactional leadership. J Appl Psychol 88(2): 207-18

[8]. Northouse PG (2010) Leadership: Theory and Practice. 5th edn. Sage Publications, London

[9]. Ilies R, Curseu PL, Dimotakis N, Spitzmuller M (2012) Leaders' emotional expressiveness and their behavioural and relational authenticity: effects on followers. European J Work Organizational Psychology, 17 January 2012. http://dx.doi.org/10.1080/1359432X.2011.626199 (accessed 29 October 2012)

[10]. Casida J, Parker J (2011) Staff nurse perceptions of nurse manager leadership styles and outcomes. J Nurs Manag 19(4): 478-86

[11]. Wilmot, W. W., \& Hocker, J. L. (2001). Interpersonal conflict. New York, NY: McGraw-Hill.

[12]. Hendel T., Fish M., \& Galon V., (2005). Leadership style and choice of strategy in conflict management among Israeli nurse managers in general hospitals. Journal of Nursing Management, 2005, 13, 137-146

[13]. Fakhimi, F, (2004), Organizational Behavior Management, No.1, Tehran Hastan Press

[14]. DeChurch, L. A., \& Marks, M. A. (2001). Maximizing the benefits of task conflict: The role of conflict management. International Journal of Conflict Management , 12: 4-22.

[15]. Allred, C., Arford, P. H., Michel, Y., Veitch, J. S, Dring, R. \& Carter, V. (1995). Case management: The relationship between structure and environment. Nursing Economics, 13(1), 32-55.

[16]. Shirey MR. (2009). Authentic leadership, organizational culture, and healthy work environments. Crit Care Nurse Q. 32:189-198.

[17]. Clark-Burg K., (2013). Juggling multiple priorities to please: A grounded theory of nurse managers' process of decision making. Theses. Paper 77. available at: http://researchonline.nd.edu.au/theses

[18]. Carroll, P. (2008). Nursing leadership and management: A practical guide. Sydney: Cengage Learning.Cathcart, E. B., Greenspan, M. \& Quin, M. (2010). The making of a nurse manager: The role of experiential learning in leadership development. Journal of Nursing Management, 18(4), 440-7.

[19]. Tatum, B.C., Eberlin, and Kottraba. (2003). Leadership, decision making and organizational justice. Management Decision, 41(10), 1006-1016.

[20]. Jasmin H. (2007). Effective decision making: Topic Gateway Series No. 40 Technical Information Service December. 
[21]. Johnson, D.W., and Johnson, F.P. (2000). Joining together: Group theory and group skills, 7th ed. Boston: Allyn and Bacon.

[22]. James O \& Ogbonna I.G. (2013). Transformational vs. Transactional Leadership Theories: Evidence in Literature. International Review of Management and Business Research. Vol. 2 Issue.2, pp, 355-61.

[23]. Harvey J. \& Technical Information Service. (2007). Effective decision making. Topic Gateway Series No. 40. available at: www.cimaglobal.com

[24]. Kalf H. (2010). The Relationship between Transformational Leadership and the Creative Management among Academics Heads Departments at the Islamic University in Gaza. Published Master Thesis. Faculty of Commerce, Islamic University.

[25]. Rahim M. A. \&. Magner, N. R. (1995), Journal of Applied Psychology, 80(1), 122-132. In W. Wilmot and J. Hocker (2011), Interpersonal Conflict (pp. 146-148). New York: McGraw-Hill. Used with permission of publisher.

[26]. Abahe (2011). Factors influencing the administrative decision-making in healthcare organizations. Available at: www.abahe.co.uk/Research.../decision-making-in-health-organizations.p.

[27]. El-Ghazali H (2012). The Impact of Transformational Leadership on Decision Taking Process Effectiveness in the Jordanian Insurance Companies. Published Master thesis. Middle East University, College of Business. Jordan.

[28]. Radstaak, K. (2008). Training and coaching of transformational leadership. Published PhD. Faculty of Arts .Wilhelm University.

[29]. Tourangeau, A., \& McGilton, K. (2004). Measuring leadership practices of nurses using the leadership practices inventory. Nursing Research, 53(3), 182-189.

[30]. Danish, K.Q. Munir, Y. Nazir, S. Abbasi, H \& Hunbal, H. (2013). Effect of Knowledge Sharing, Participative Decision Making and Transformational Leadership on Organizational Performance. World Applied Sciences Journal. 24 (10): 1339-1347.

[31]. Nguyen, N, H. \& Mohamed, S. 2011. Leadership behaviors, organizational culture and knowledge management practices: An empirical investigation. Journal of Management Development, pp: 206-221.

[32]. Liu, K. L., Chang C. C. \& Hu, L. 2010. Exploring the effects of task characteristics on knowledge sharing in libraries. Library Review, pp: 455-468.

[33]. Zori, S. \& Morrison, B. (2009). Critical thinking in nurse managers. Nursing Economics, 27(2), 75-80, 98.

[34]. Cummings, G., Lee, H., Macgregor, T., Davey, M., Wong, C., Paul, L. \& Stafford, E. (2008). Factors contributing to nursing leadership: A systematic review. Journal of Health Services Research \& Policy, 13(4), $240-8$.

[35]. Byram, D. (2000). Leadership: A skill, not a role. Advanced Practice in Acute Critical Care, 11(3).

[36]. Fitzpatrick, C. (2007). Training methods and tactical decision making simulations. Master's Thesis. Naval Postgraduate School. USA

[37]. Brooks, B., \& Anderson, M. (2004). Nursing work life in acute care. Journal of Nursing Care Outcome, 19(3), 269-275.

[38]. Tourangeau, A. (2003). Building nurse leader capacity. Journal of Nursing Administration, 33(12), 624-626.

[39]. Wicker, L.T. (2008). Self-report of nursing leadership practice after completion of training. Published PhD of philosophy. College of nursing. University of Arizona

[40]. Kelloway, E.K., Barling J., \& Helleur J. (2000): Enhancing transformational leadership: the role of training and feedback. The Leadership and Management Development Journal, Vol. 21 No.3, pp. 145-9.

[41]. Modassir, A. \& Singh, T. (2008). Relationship of Emotional Intelligence with Transformational Leadership and Organizational Citizenship Behavior. International Journal of Leadership Studies, Vol. 4 Iss. 1, pp. 3-21

[42]. Dionne, S., Yammarino, F., Atwater, L., \& Spangler, W. (2004). Transformational leadership and team performance. Journal of Organizational Change Management, 17(2), 177193.

[43]. Zohar, D. and Tenne-Gazit, O. (2008), "Transformational leadership and group interaction as climate antecedents: a social network analysis", Journal of Applied Psychology, Vol. 93 No. 4, pp. 744-57.

[44]. Leary A.M. (2011). The implementation of conflict management training into the post anesthesia care setting for staff nurses during yearly competency day. Published Master thesis. Office of Graduate Studies. University of Massachusetts Boston

[45]. Copley, R.D. (2008). Conflict management styles: a predictor of likeability and perceived effectiveness among subordinates. Published Master thesis. Faculty of the University Graduate School. Indiana University

[46]. Anthony MK, Standing TS, Glick J, Duffy M, Paschall F, Sauer MR, Sweeney DK, Modic MB, Dumpe ML. (2005). Leadership and nurse retention: The pivotal role of nurse managers. Journal of Nursing Administration, 35:146-155.

[47]. Welford C. (2002) Matching theory to practice. Nursing Management 9 (4), 7-11.

[48]. Brinkert R. (2011). Conflict coaching training for nurse managers: a case study of a two-hospital health system. Journal of Nursing Management, 19:80-91.

[49]. Deans C. (2004). The effectiveness of a training program for emergency department nurses in managing violent situations. Australian Journal of Advanced Nursing, 21:17-22.

[50]. Pavlakis A, Kaitelidou D, Theodorou M, Galanis P, Sourtzi P, Siskou O. (2011). Conflict management in public hospitals: the Cyprus case. International Nursing Review, 58:242-248.

[51]. Rahim, A. (2002). Toward a theory of managing organizational conflict. The International Journal of Conflict Management. Vol. 13, No. 3, pp. $206 \square 235$

[52]. Kaitelidou D, Kontogianni A, Galanis P, Siskou O, Mallidou A, Pavlakis A, Kostagiolas P, Theodorou M, Liaropoulos L. (2012). Conflict management and job satisfaction in pediatric hospitals in Greece. Journal of Nursing Management, 20:571-578.

[53]. Mahon MM \& Nicoreta AM. (2011). Nursing and conflict communication: avoidance as preferred strategy. Nursing Administration Quarterly, 35:152-163.

[54]. Thompson, C. McCaughan, D. Cullum, N. Sheldon, T. Thompson, D. Mulhall, A. (2002). Nurses' Use of Research Information in Clinical Decision Making: A Descriptive and Analytical Study. Report Presented to the NHS R\&D Programme in Evaluating Methods to Promote the Implementation of R\&D.

[55]. Niekerk V. (2005). The implementation of effective decision making strategies by secondary school principals in the Free State Province. Faculty of Education. Free State Province. University of South Africa

[56]. College of Nurses of Ontario. (2010). Quality Assurance Overview. Unpublished Manuscript.

[57]. Effken, J., Verran, J., Logue , M. \& Hsu, YC. (2010). Nurse Managers' Decisions: Fast and Favoring Remediation. JONA. Volume 40, Number 4, pp 188-195.

[58]. Dietrich C. (2010). Decision Making: Factors that Influence Decision Making, Heuristics Used, and Decision Outcomes. The International Student Journal. VOL. 2 NO. 02 |PG. 1/3|

[59]. Tanner, C. (2006).Thinking like a nurse: A research based model of clinical judgment in nursing. Journal of Nursing Education, 45(6), 204-212.

[60]. Gaskin, C. J., Ockerby, C. M., Smith, T. R., Russell, V. \& O'Connell, B. (2012). The challenges acute care nurse unit managers face and the strategies they use to address them: Perceptions of directors of nursing and nurse unit managers. Journal of Management \& Organization, 2393-2427. 
[61]. Ahmed, M., \& Safadi, E. (2013). Decisional involvement among Nurses: Governmental versus Private Hospital. Health Science Journal. Volume, 7 , issue 1

[62]. Bord Altranais (2000) Guidance to Nurses and Midwives on the Development of Policies, Guidelines and Protocols. A Bord Altranais, Dublin.

[63]. Michel, L. (2007). Understanding decision making in organizations to focus its practices where it matters. Measuring Business Excellence, 11, 33-45. Retrieved August 21, 2007, from ProQuest database.

[64]. Lorber, M. \& Skela Savic, B. (2011). Perceptions of managerial competencies, style, and characteristics among professionals in nursing. Croatian Medical Journal, 52(2), 198-204.

[65]. Ellis JR.\& Hartley CL.( 2005). Managing and coordinating nursing care. (4th ed.). Eds Lippincott Williams \& Wilkins, Philadelphia.

[66]. Cherie A.,\& Gebrekidan A.B. (2005). Nursing Leadership and Management. Ethiopia Public Health Training Initiative. Produced in collaboration with the Ethiopia Public Health Training Initiative, The Carter Center, the Ethiopia Ministry of Health, and the Ethiopia Ministry of Education.Pp.72-76.

[67]. Nutt, P. C. (2006). Comparing public and private sector decision-making practices. Journal of Public Administration Research and Theory, 16, 289-318. Retrieved August 24, 2007, from ProQuest database.

[68]. Benner P, Tanner C, \&Chesla C. (1996). Expertise in Nursing Practice: Caring, Clinical Judgment and Ethics. Eds., Springer, New York,

[69]. McGuire, E., \& Kennerly, S. (2006). Nurse managers as transformational and transactional leaders. Nursing Economics, 24(4), 179-185.

[70]. Pielstick, D. C. (1998). "The Transforming Leader" A Meta-Ethnographic Analysis. The Community College Review. December.

[71]. Cruz, M.G., Henningsen, D.D. \& Smith, B.A. (1999), "The impact of directive leadership on group information sampling, decisions and perceptions of the leader", Communication Research, Vol. 26 No. 3, pp. 349-69. 